\title{
Study on risk zoning of urban population based on Vulnerability
}

\author{
Y. GAO \& T. CHEN \& H.Y. YUAN \& Q.Y. HUANG \\ Institute of Public Safety Research, Department of Engineering Physics, Tsinghua University, Bei- \\ jing, China
}

KEYWORD: City Earthquake; Shichahai Street; Population vulnerability; Risk zoning map

ABSTRACT: In order to response to the great threat and loss of cities brought by earthquake, through the research of Shichahai street communities population vulnerable, established the corresponding evaluation system and model, drawn the risk zoning map. The results show that the community of Shichahai street has its own vulnerability, and the population vulnerability of the community has a lot of differences.

\section{Earthquake Vulnerability of city}

\section{Status quo of vulnerability research}

City is the strong coupling of human - object - the function system, urban vulnerability analysis should not only considering anti disaster ability of all kinds of building / structure, also need to consider the layout of the urban population and behavior characteristics in the disaster. Beijing is one of the earthquake areas in the eastern part of China, often occurs a moderate scale of earthquake, although basically does not cause or causes only a small damage and loss, but the earthquake caused tremendous social impact. Therefore, in the Beijing area, the earthquake disaster is one of the important risk sources of the public incident.

Once the city has an earthquake, any link out of control will have a devastating consequences. Therefore, only by fully understanding the vulnerability of the city, can it provide reliable basis for the city earthquake disaster reduction. Vulnerability is usually the damage of the bearing bodies under the earthquake action. The characteristics of China's urban development and urban disaster, has great significance to our country urban safety and social sustainable development to establish a sound evaluation index of urban disaster emergency capability, is the priority of our country urban safety and disaster prevention at present.

The population vulnerability assessment of the earthquake disaster, whether in earthquake emergency or in the economic and social development plan, is a very important content. Casualties were mainly due to the collapse of housing, in general, the casualties are large relatively in the areas of the dense housing construction and the population density. That is, the population vulnerability level is high in the residential land, public facilities, industrial land and special land.

The emergency management division of urban earthquake disaster is based on microscopic regions of a disaster emergency of management idea, the division will make pre disaster emergency preparedness, disaster emergency rescue, post disaster emergency recovery and actual emergency management operation in the local area, the emergency management work closer to the people (the victims in disaster) and easier for local potential hazard assessment and disaster accident emergency rescue and management, easier to disaster response infrastructures for assessment and construction, also are more likely to promote public disaster consciousness education and disaster emergency rescue exercises.

Urban vulnerability analysis and risk evaluation system is an important support to promote the urban social stability and economic development, protect the people live and work, maintain the normal operation of the national management system. Pay attention to the key technology of the world, such as the monitoring and controlling, simulating analysis, forecasting and warning, emergency disposal and comprehensive evaluation of the city vulnerability.

United States did the integrated risk assessment on 10 kinds of natural disasters, such as floods, tsunamis, storm surges and tornadoes. Japan, Britain and other countries did the comprehensive evaluation of typhoons, floods, tsunamis and other disasters. The Federal Emergency Management Agency (FEMA) and the National Academy of Building Sciences (NIBS) jointly developed a risk assessment software system (HAZUS) for earthquakes, floods and hurricanes. Comprehensive consideration of all kinds of disasters that the city is facing, to study the urban vulnerability analysis technology re- 
search and development of urban risk evaluation system, to provide comprehensive risk awareness and decision-making basis for urban management, which is the important development trend of international risk assessment techniques.

\section{Typefont, typesize and spacing}

The city vulnerability has the following characteristics: (1) due to the aging of the city system, strengthening, update ceaselessly, city system is not static, but evolve in time and space, but this is likely to evolve a higher level of development; (2) when the subsystem by the direct impact of a disaster sent the interference signal, the remaining subsystems disturbed by the interference of interference factor will be given feedback over a period of time, on its interference reaction showed a delay; (3) one of the subsystems, suffered a sudden incident, because of the correlation among the city's internal system, so that it can quickly transfer damage capability to other subsystems, this is the real reason that the city vulnerability is dangerous.

\section{The population vulnerability of urban earthquake}

In order to reduce the degree of urban vulnerability, the city should strengthen the response to disasters and the disaster management ability of cities. At present, the trend of the development of international disaster management is risk management instead of disaster management. There are some domestic scholars for the vulnerability of urban disaster, defined the evaluation method of regional vulnerability assessment, determined the vulnerability index of regional disaster body.

Different vulnerability definitions have different evaluation index systems and evaluation methods. From the point view of the current research situation of domestic and foreign, vulnerability definition has three categories: the first category, with the index of hazard affected body to reflect the characteristics of hazard affected body; common features of another definition is combine the natural phenomenon with social consequences, or that is a function of the two interassociation systems, or they are superimposed on each other. The third category, to define the concept of vulnerability with the social consequences of natural phenomena.

\section{Data standardization processing}

Make the attribute data standardization with the maximum and minimum values, as follows:

$$
Y_{i j}=\frac{\mathrm{X}_{i j}-\min \left(X_{j}\right)}{\max \left(X_{j}\right)-\min \left(X_{j}\right)}
$$

Where, $Y_{i}=$ the value after sequence standardization between the unit $\mathrm{i}$ and the index factor $\mathrm{j} ; \max \left(X_{i}\right)=$ the maximum of the index $\mathrm{j}$ in the total units; and $\min \left(X_{i}\right)=$ the minimum of the index $\mathrm{j}$ in the total units.

\section{Comprehensive evaluation of population vulnerability}

Based on the standardized data and the weight obtained by the expert scoring method and the analytic hierarchy process, calculated the population vulnerability index of each unit, the concrete methods are as follows:

$$
M_{i}=\frac{\sum_{i=1}^{n}\left(Y_{i j} \times K_{j}\right)}{\sum K_{j}}
$$

Where, $M_{i}=$ the population vulnerability index of the unit $\mathrm{i}$, the greater the index, the stronger the population fragility, and vice versa; $K_{j}=$ the corresponding weight of the index factor j, which is obtained with the expert scoring and AHP; and $Y_{i j}=$ the value after sequence standardization between the unit $i$ and the index factor $j$.

\section{Typical case studies}

Shichahai street is located in the northeast of Xicheng District. Through analyzing the data of Shichahai street community, get the community basic population distribution, distribution of the female population proportion and the aging population, as shown in Figure 1 and Figure 2. 


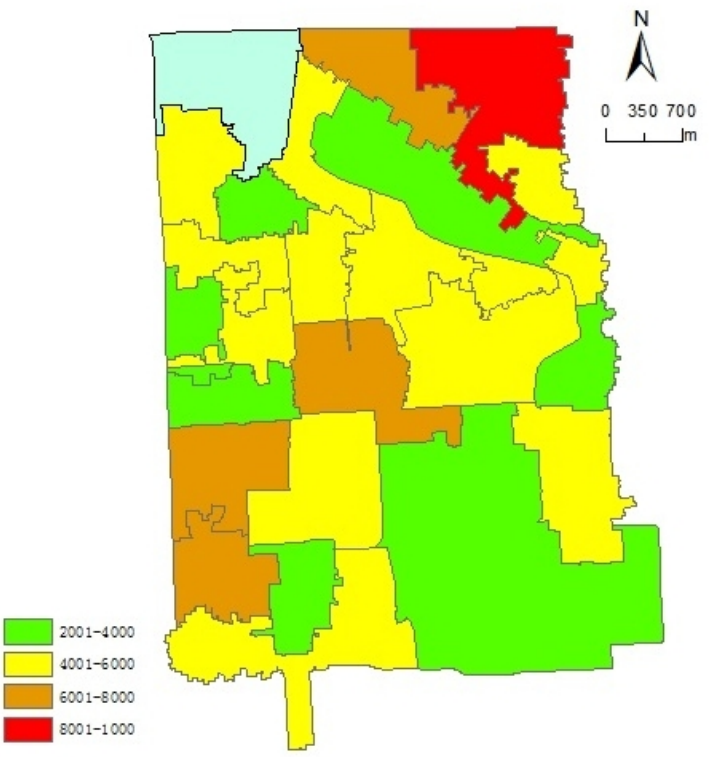

Figure 1. The basic population distribution of Shichahai street community

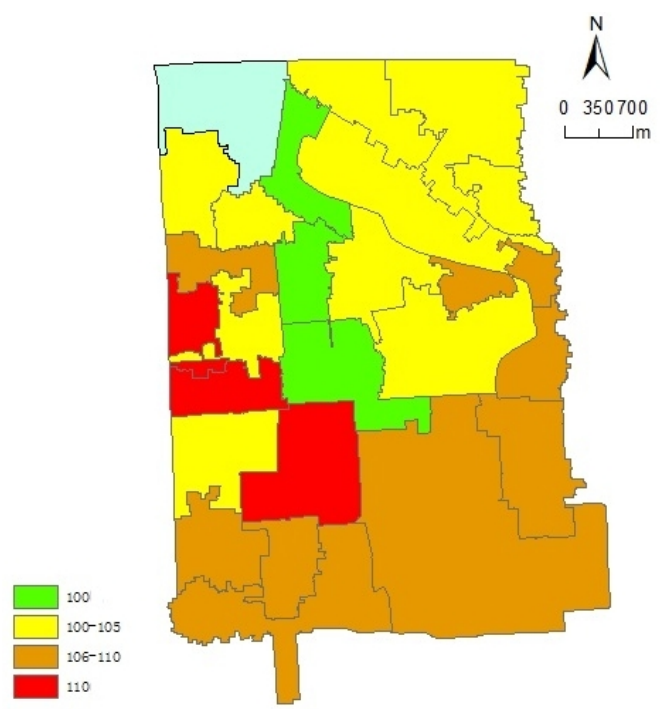

(a) female population

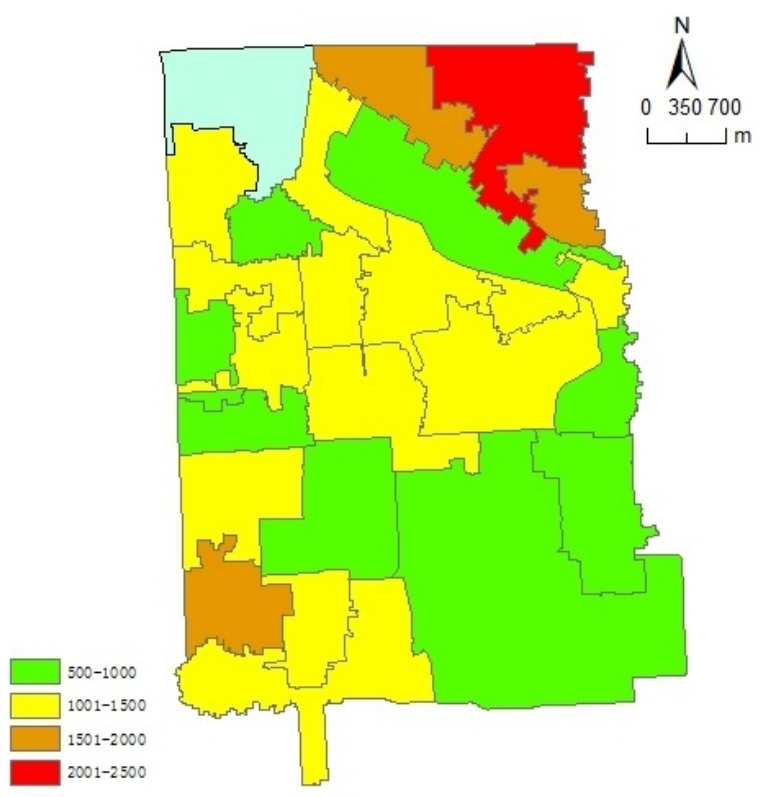

(b) ageing population

Figure 2. The spatial distribution of major demographic indicators in Shichahai street community 
To carry out standardized treatment and comprehensive evaluation for the above factors, obtained the population vulnerability zoning map of Shichahai street community, as shown in Figure 3.

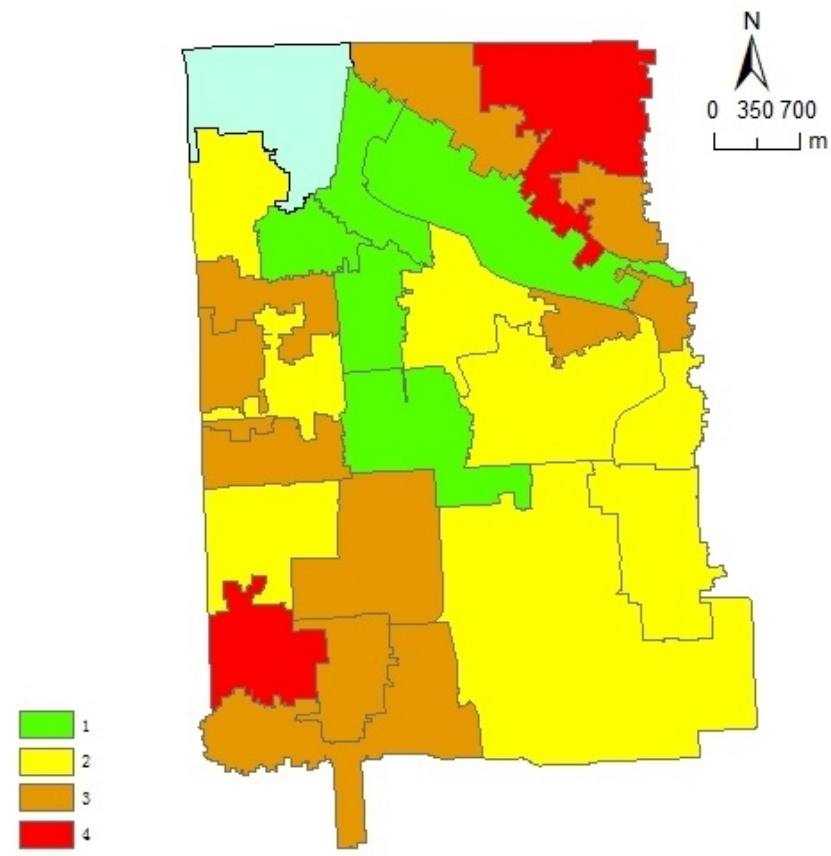

Figure 3. The population vulnerability of Shichahai street community

From the picture, it can be seen that the population of the community in northeast and southwest of Shichahai is vulnerable. The population in the southwest is not large, but the proportion of female population is large, which leads to the high vulnerability of population. The population distribution in the northeast is large, and the ageing population is more, so the overall vulnerability is higher. The population of other communities is small, the proportion of female and ageing population is relatively low, so the vulnerability population is relatively low.

\section{conclusions}

Through analyzing the vulnerability characteristics of the urban earthquake, established the evaluation model of population vulnerability, and analyzed the vulnerability of the communities in Shichahai street, drawn the vulnerability risk zoning map of communities. In the risk zoning map, the vulnerability area can be displayed visually, so that the usual emergency management can be easily, and can take the effective preventive measures in the emergency. Targeted prevention and governance can get more time to rescue, which will largely reduce the occurrence of casualties and property damage in the earthquake.

\section{REFERENCES}

[1] Li, Z. \& Wang, X.Q. 2010. The overview of rapid and blind evaluation of seismic damage with micro and macro methods. Earthquake (2): 134-142.

[2] Li, J. \& Jiang, J.H. 2000. Risk analysis of secondary fire in urban earthquake. Journal of natural disasters 9 (2): 87-92.

[3] Pan, M. \& Jin, J. \& Xu, Y.R. 2007. Risk assessment of urban seismic disasters. Inland earthquake (02): 135-141.

[4] Tie, Y.B. \& Tang, C. 2005. The evaluation index system of urban disaster emergency response capability. Urban problem (06): 78-81.

[5] Wang, F. \& Jiang, J.Q. 2005.. The comprehensive vulnerability analysis for urban seismic disasters. Earthquake research (01): 95-101.

[6] Yang, F.P. \& Huang, C.F. 2006. The concept of urban earthquake disaster emergency management zoning. Journal of natural disasters (01): 45-51. 